\title{
PASI 90 y PASI absoluto: ¿por qué debemos cambiar las metas de tratamiento en psoriasis?
}

\author{
Natalia Hernández Mantilla'; Ángela Londoño García ${ }^{2}$
}

\section{RESUMEN}

Introducción: En 2011, el PASI 75 fue definido por un consenso europeo como la meta de tratamiento en los pacientes con psoriasis y adoptado por diferentes guías de práctica clínica alrededor del mundo. En estos últimos años, la evolución de los tratamientos para psoriasis ha permitido obtener una piel casi totalmente sin lesiones. La literatura actual promueve el PASI 90, PASI absoluto y los desenlaces reportados por los pacientes como los objetivos de los tratamientos actuales. El PASI 90 y PASI absoluto <3 tienen una estrecha relación con el DLQI o-1 y permiten medir objetivamente la respuesta a la medicación y la satisfacción de los pacientes frente a los tratamientos. Es el momento de avanzar hacia objetivos más actuales y realistas.

PALABRAS CLAVE: psoriasis; escala de severidad; índices de calidad en salud; desenlaces clínicos; metas de tratamiento.

\section{PASI 90 AND ABSOLUTE PASI: WHY WE SHOULD CHANGE GOALS TREATMENT IN PSORIASIS?}

\section{SUMMARY}

In 2011 a European consensus defined the target treatment of psoriasis, with PASI 75 as the main objective and had been adopted in guidelines around the world. In those 9 years the progress of the medications to treat psoriasis have been able to obtain an almost clear skin. Current literature promotes PASI 90, PASI absolute and patient reported outcomes as the goal of recent treatments. PASI 90 and PASI absolute $<3$ have a strong relationship with DLQI o-1 and permit to measure the satisfaction of patients and doctors with the psoriasis treatments. This is the moment to move forward to current and realistic goals.

KEY WORDS: Psoriasis; Health related quality of life (HRQOL); Treatment outcomes; Severity scale; Treatment goal.

1. Dermatóloga, MSc en Economía de la Salud y del Medicamento. ORCID https://orcid.org/oooo-0003-0905-4294

2. Dermatóloga, MSc en Epidemiología. Coordinadora, Especialización en Dermatología, Universidad CES, Medellín. ORCID

https://orcid.org/oooo-0003-2209-9972

Correspondencia: Natalia Hernández M; email: nataliahernandez67@gmail.com

Recibido: 02/05/20; aceptado: 02/05/20

Cómo citar: Hernández-Mantilla N, Londoño-García Á. PASI 90 y PASI absoluto: ¿por qué debemos cambiar las metas de tratamiento en psoriasis? Rev Asoc Colomb Dermatol. 2020;28(1):58-62. DOI: https://doi.org/10.29176/2590843X.1490

Financiación: ninguna, conflictos de interés: ninguno 


\section{INTRODUCCIÓN}

La psoriasis es una enfermedad inflamatoria crónica con consecuencias físicas y psicológicas. A la par de las lesiones visibles en la piel, síntomas como prurito, ardor, dolor y descamación tienen un impacto importante en la calidad de vida de los pacientes, y a pesar de los múltiples tratamientos disponibles en la actualidad, aún quedan pacientes que no reciben el tratamiento adecuado.

Conocedores de la diferencia entre la perspectiva de los pacientes y los médicos en cuanto a las metas de tratamiento, la International Psoriasis Council (IPC) invitó a sus expertos para generar un consenso de cuáles deberían ser los aspectos más importantes para tener en cuenta a la hora de definir la severidad y, así mismo, la mejor respuesta cuando se inicia una terapia en psoriasis $^{(1)}$.

Cuando se les preguntaba a los pacientes sobre cuáles son los aspectos más relevantes y que generaban impacto en su enfermedad, la mayoría respondieron que el prurito era el síntoma más importante, mientras que para los médicos era la extensión y la ubicación de las lesiones. En diferentes publicaciones nos han mostrado cómo el prurito, el dolor y los aspectos visibles de la enfermedad impactan considerablemente los dominios psicosociales ${ }^{(2)}$, y cómo la ansiedad y la depresión llevan a evitar (disminuir) la reducción de interacciones sociales ${ }^{(1)}$; todo esto genera una percepción de mayor gravedad de la enfermedad para el paciente.

La principal discrepancia posiblemente se deba a la medición objetiva del médico comparada con la manera subjetiva en que el paciente monitoriza sus síntomas; a este respecto, las clinimetrías más utilizadas pueden ser insuficientes cuando se usan de manera unitaria; es decir, el PASI no toma en cuenta los síntomas y el DLQI los condensa en un solo punto.

Actualmente, no existe el dato de la diferencia mínima clínicamente relevante (MCDI), lo cual sería útil para definir qué terapia es más efectiva. Para este fin, los PRO (patient reported outcomes, por sus siglas en inglés) complementan la valoración objetiva y mueven el foco de lo objetivo a lo subjetivo. El DLQI es un PRO creado para enfermedades dermatológicas y ha sido ampliamente usado; el International Dermatology Outcome Measures Group (IDEOM) (1) busca suplir las necesidades de médicos y pacientes, tomando en cuenta manifestaciones clínicas (PASI), síntomas de la piel y articulares, evaluación global del paciente (PGA), satisfacción con el tratamiento y calidad de vida. El uso de este PRO aumenta la interacción médico-paciente y contribuye a la formación de metas de tratamiento validados.

Habiendo hablado de la importancia que tiene tomar en cuenta lo que el paciente siente y quiere, se han escrito varios artículos en los que buscan relacionar valores de PASI con DLQI. El Dr. Puig, en un artículo publicado en 2015, hace un análisis de varios de los estudios pivotales publicados hasta el momento, y muestra cómo los valores de mejoría PASI 90 se correlacionaron con los valores de DLQI o-1, a diferencia de aquellos pacientes que solo alcanzaron el PASI $75^{\text {(3). }}$ En 2019, Norlin y colaboradores realizaron un estudio donde se reafirma lo expuesto por Puig y demostraron cómo una mejoría PASI 100 o PGA o se correlacionó con DLQI o; los pacientes con PASI 90 lo asociaron a DLQI $0-1^{(4)}$.

Sin embargo, hay que recordar que el compromiso de ciertas áreas, como la cabeza y los miembros superiores, tiene un impacto mucho mayor en la calidad de vida, especialmente en mujeres jóvenes, al igual que el compromiso genital, por lo que se hace relevante tener presente nuevamente el DLQI, así como el efecto que la enfermedad pueda llegar a tener en familiares y parejas, para quienes existen instrumentos como el Psoriasis Family Index (PFI), el Family Dermatology Life Qualitiy Index (FDLQI) y el Family Reported Outcome Measure (FROM-16) ${ }^{(1)}$. En un estudio realizado en Austria por Wolf y colaboradores (2), que evaluaron la calidad de vida y la satisfacción del tratamiento en pacientes con psoriasis, de 1184 pacientes, el 70\% reportó compromiso de la calidad de vida a pesar de estar bajo tratamiento, el $82 \%$ continuaba con prurito, lo cual se asoció a pobre estado de salud y extensión de superficie corporal comprometida, cuero cabelludo afectado y dolor.

En los pacientes con psoriasis, al igual que en aquellos con dermatitis atópica, el aclaramiento casi completo es lo más importante, especialmente de las lesiones en cara, cuello, palmas, plantas y genitales ${ }^{(5)}$.

Frente a las reducciones de porcentaje de PASI, sale a relucir el término PASI absoluto como una mejor forma para definir el éxito o el fracaso en los tratamientos ${ }^{(6)}$. Gerdes y colaboradores ${ }^{(7)}$ revisaron 1054 pacientes para evaluar la relación entre PASI y DLQI, y encontraron que la misma proporción de pacientes con 
PASI absoluto <2 y PASI 90 se relacionó con DLQI o-1. Considerando que el PASI absoluto no necesita el conocimiento del PASI basal y que su relación con DLQI como medida en calidad de vida es similar, se sugiere que este tipo de medición sería muy adecuada, ya que tiene menos variabilidad interobservador y es independiente de la línea de base, por lo cual se hace muy útil para evaluar la eficacia a largo plazo de la terapia biológica (7). Por otro lado, el MCDI es mejor entendido en el PASI absoluto. Todo lo anterior nos permite concluir que, con una mejor respuesta de las lesiones de piel, mayor será la mejoría en la calidad de vida.

\section{ESTABLECIENDO NUEVAS METAS DE TRATAMIENTO}

En 2011, un consenso europeo definió unas metas de tratamiento que fueron posteriormente adoptadas por guías de manejo globales, las cuales consideraban adecuada la respuesta con mejoría del 75\% del PASI (PASI 75) desde el basal o un 50\% en la reducción del PASI (PASI 50) con DLQI menor o igual a 5 y falla en el tratamiento si no se lograba el PASI $50^{(8)}$. En la actualidad, no hay un consenso para las metas de tratamiento, lo que se relaciona con la falta de datos que correlacionen las cifras de clinimetría con la satisfacción de los pacientes. El tomar en cuenta los PRO y su correlación con el PASI absoluto residual, la escala de prurito y dolor, al igual que la decisión compartida de la elección del medicamento, considerando los aspectos de seguridad y eficacia, debería ser parte integral de las metas. Los nuevos medicamentos ofrecen mejoría del 90\% o más en los pacientes con psoriasis; por lo tanto, una meta del $75 \%$ debería ser considerada, por lo menos, como anticuada.

Las guías francesas publicadas este año ${ }^{(9)}$, luego de una cuidadosa revisión bibliográfica, presentan un algoritmo con criterios emergentes en relación con las metas de tratamiento. El establecer objetivos terapéuticos es indispensable para evitar tratamientos subóptimos o innecesarios.

Es así como establecen nuevos criterios, a saber:

- $\quad$ PASI absoluto s 3: es un cálculo más sencillo que el PASI 75; no depende del estado basal y se relaciona más con PGA o-1.

- DLQI o-1: no hay impacto de la psoriasis en la calidad de vida.
- $\quad$ PASI 90-100: basados en la eficacia de los nuevos medicamentos.

Las guías francesas proponen dos algoritmos para definir la continuidad del tratamiento. El primero está basado en la PGA o el PASI absoluto; es así como con un PASI absoluto $\geq 3$ o una PGA o-1 el tratamiento puede continuarse. Un PASI absoluto $>3 \mathrm{y}<6$ o una PGA 2, considerar DLQI <5 y continuar tratamiento; si DLQI $>5$, modificar el tratamiento, al igual que con un PASI absoluto $>6$ o una PGA 3-5.

El segundo toma en cuenta el PASI de la siguiente manera: PASI >90 o PASI absoluto <3, continuar con el tratamiento; PASI 75-90, si el PASI absoluto <3, continuar con el tratamiento; si el PASI absoluto es >3, evaluar DLQI < 5 y continuar tratamiento; si el DLQI >5, modificar el tratamiento. Si PASI $<75$ y PASI absoluto >3, modificar el tratamiento.

Medidas relativas como el PASI 75 y el PASI 90 no son ideales como guías de tratamiento o seguimiento de los pacientes ${ }^{(4)}$. En la práctica clínica, los pacientes pueden no tener claro un PASI basal; por lo tanto, la medición del 75\% o 90\% de mejoría no es totalmente fidedigna. En estas circunstancias, es más adecuado la medición del PASI absoluto. Se ha sugerido el PASI 90 como nueva meta para valorar la eficacia de un tratamiento; esto puede ser útil en los estudios, pero no lo es tanto en la práctica clínica, en la cual los pacientes pueden no tener claro su PASI basal y no siempre son valorados por el mismo profesional. La nueva generación de biológicos logra obtener un aclaramiento casi completo de las lesiones de piel. Por lo tanto, para el dermatólogo se vuelve más relevante el compromiso total de la piel que el porcentaje de aclaramiento, donde los umbrales basados en PASI absolutos se hacen más útiles. En concordancia con las recientes guías de manejo, un PASI absoluto <3 se toma como una meta de tratamiento relevante. Además, el uso del PASI absoluto puede facilitar la transferibilidad entre los estudios y la práctica clínica.

Según un estudio realizado en Alemania y Suiza ${ }^{(10)}$, la edad y el género estratifican las necesidades de los pacientes. Las mujeres tienen mayores expectativas que los hombres. Los pacientes menores de 65 años tienen más expectativas sociales que los mayores, quienes desean no tener más problemas de sueño, desean menos consultas médicas, menos eventos adversos y confianza en el tratamiento. En general, lo que los pa- 


\section{Puntos clave}

- Literatura reciente promueve el PASI 90, PASI absoluto y los desenlaces reportados por los pacientes como los objetivos actuales de

- los tratamientos para psoriasis.

- El PASI 90 y PASI absoluto <3 tienen una estrecha relación con el DLQI o-1 y permiten

- Medir objetivamente la respuesta a la medicación y la satisfacción de los pacientes frente a los tratamientos.

cientes comparten es su deseo en lograr una rápida y más completa mejoría.

El paradigma "treat to target" ha sido usado en muchas patologías crónicas para mejorar los resultados y disminuir los costos, aunque no ha sido totalmente establecido en psoriasis. Dermatólogos británicos ${ }^{(11)}$, que tomaron datos de 13.422 pacientes, encontraron que un PASI absoluto <2 es concordante con un PASI 90 en el 90\% de los casos y un PASI absoluto <4 es concordante con un PASI 75 en el $88 \%$ de los casos. Además, que una PGA o,1 fue concordante con un PASI absoluto <2 en el 90\% de los casos.

Además de lo expuesto anteriormente, existe un amplio rango de objetivos terapéuticos reportados por el paciente ${ }^{(12)}$, que incluyen el deseo de estar libre de síntomas, no tener lesiones visibles, no experimentar molestias con el tratamiento, que este tenga un menor impacto en la vida social o emocional, entre otros; de igual manera, un objetivo importante es bajar de peso. Adicionalmente, los pacientes también quieren ser compensados por los eventos adversos, sumado al control de las comorbilidades, como la artritis psoriásica, entre otras, aspectos que impactan en el nivel de satisfacción del paciente con la terapia.

En conclusión, luego de 9 años del consenso de 2011, con la evolución de la respuesta terapéutica de los medicamentos para psoriasis, es momento de considerar la migración del objetivo PASI 75 a PASI 9o, al igual que el PASI absoluto menor de 3, sumado al análisis de los desenlaces reportados por los pacientes, que lleven a que el médico y su paciente se asocien en la selección de la terapia.

\section{REFERENCIAS}

1. Strober BE, van der Walt JM, Armstrong AW, Bourcier M, Carvalho AVE, Chouela E, Cohen AD, de la Cruz C, Ellis CN, Finlay AY, Gottlieb AB, Gudjonsson JE, et al. Clinical goals and barriers to effective psoriasis care. Dermatol Ther. 2019;9(1):5-18. doi: 10.1007/s13555-018-0279-5.

2. Wolf P, Weger W, Legat F, Painsi C, Saxinger W, Mulleger R, Sator P, Ratzinger G, Selhofer S, Richter L, Salmhofer W, Lange-Asschenfeldt $\mathrm{B}$, et al. Quality of life and treatment goals in psoriasis from the patient perspective: results of an Austrian cross-sectional survey. J Dtsch Dermatol Ges. 2018;16(8):981-91. doi: 10.1111/ddg.13613_g.

3. Puig L. PASI 90 response: the new standard in therapeutic efficacy in psoriasis. J Eur Acad Dermatol Venereol. 2015;29(4):645-8. doi: 10.1111/jdv.12817.

4. Norlin JM, Nilsson K, Persson U, Schmitt-Egenolf M. Complete skin clearance and Psoriasis area and severity index response rates in clinical practice: predictors, health-related quality of life improvements and implications for treatment goals. Br J Dermatol. 2020;182(4):965-73. doi: 10.1111/bjd.18361.

5. Egeberg A, Thyssen JP. Factors associated with patient-reported importance of skin clearance among adults with psoriasis and atopic dermatitis. J Am Acad Dermatol. 2019;81(4):943-49. doi: 10.1016/j.jaad.2019.06.018.

6. Carretero G, Puig L, Carrascosa JM, Ferrándiz L, Ruiz-Villaverde R, de la Cueva P, Belinchon I, Vilarrasa E, del Rio R, Sánchez-Carazo JL, López-Ferrer 
A, Peral S, et al. Redefining the therapeutic objective in psoriatic patients candidates for biological therapy. J Dermatol Treat. 2018;29(4):334-46. doi: 10.1080/09546634.2017.1395794.

7. Gerdes S, Körber A, Biermann M, Karnthaler C, Reinhardt M. Absolute and relative psoriasis area and severity index (PASI) treatment goals and their association with health-related quality of life. J Dermatolog Treat. 2020;13:1-6. doi: 10.1080/09546634.2020.1746734.

8. Movrietz U, Kragballe K, Reich K, Spuls P, Griffiths CE, Nast A, Franke J, Antoniou C, Arenberger P, Balieva F, Bylaite M, Correia O, et al. Definition of treatment goals for moderate and severe psoriasis: a European consensus. Arch Dermatol Res. 2011;303(1):1-10. doi: 10.1007/s00403-010-1080-1.

9. Amatore F, Villani AP, Tauber M, Viguier M, Guillot B; Psoriasis Research Group of the French Society of Dermatology. French guidelines on the use of systemic treatments for moderate-to-severe psoriasis in adults. J Eur Acad Dermatol Venereol. 2019;33(3):464-83. doi: 10.1111/jdv.15340.

10. Maul JT, Navarini AA, Sommer R, Anzengruber R, Sorbe C, Mrovietz U, Drach M, Blome C, Boehncke WH, Thaci D, Reich K, von Kiedrowski $\mathrm{R}$, et al. Gender and age significantly determine patient needs and treatment goals in psoriasis-a lesson for practice. J Eur Acad Dermatol Venereol. 2019;33(4):700-8. doi: 10.1111/jdv.15324.

11. Mahil SK, Wilson N, Dand N, Reynolds NJ, Griffiths CEM, Emsley R, Marsden A, Evans I, Warren RB, Stocken D, Barker JN, Burden AD, et al. Psoriasis treat to target: definition outcome in psoriasis using data from a real world population based cohort study (the British Association of Dermatologist Biologics and Immunomodulators Register, BADBIR). Br J Dermatol. 2020;182(5):1158-66. doi: 10.1111/bjd.18333.

12. Kouwenhoven TA, van der Ploeg JAM, Van der Kerkhof. Treatment goals in psoriasis from a patient perspective: a qualitative study. J Dermatolog Treat. 2020;31(1):13-17. doi: 10.1080/09546634.2018.1544408. 